Mathematical Modelling and Analysis

Volume 19 Number 3, June 2014, 309-333

http://dx.doi.org/10.3846/13926292.2014.924039

(c) Vilnius Gediminas Technical University, 2014
Publisher: Taylor\&Francis and VGTU

http://www.tandfonline.com/TMMA

Print ISSN: 1392-6292

Online ISSN: 1648-3510

\title{
Existence and Numerical Approximation of Solutions of an Improved Internal Wave Model*
}

\section{Juan Carlos Muñoz Grajales}

Departamento de Matemáticas, Universidad del Valle

A.A. 25360 Cali, Colombia.

E-mail: jcarlmz@yahoo.com

Received June 18, 2013; revised May 4, 2014; published online June 1,2014

\begin{abstract}
In this paper we establish local existence of solutions for a new model to describe the propagation of an internal wave propagating at the interface of two immiscible fluids with constant densities, contained at rest in a long channel with a horizontal rigid top and bottom. We also introduce a spectral-type numerical scheme to approximate the solutions of the corresponding Cauchy problem and perform a complete error analysis of the semidiscrete scheme.
\end{abstract}

Keywords: internal waves, Cauchy problem, numerical solution, error estimates.

AMS Subject Classification: 65M70; 65M70; 35Q35; 76B55.

\section{Introduction}

In this work we derive the following system written in dimensionless variables:

$$
\begin{aligned}
& \zeta_{t}-((1-\alpha \zeta) u)_{x}=\frac{\epsilon^{2}}{6} \zeta_{x x t}, \\
& u_{t}+\alpha u u_{x}+\left(1-\frac{\rho_{2}}{\rho_{1}}\right) \zeta_{x}=\frac{\rho_{2}}{\rho_{1}} \epsilon \mathcal{H}\left(u_{x t}\right)+\frac{\epsilon^{2}}{6} u_{x x t}, \\
& \zeta(x, 0)=\zeta_{0}(x), \quad u(x, 0)=u_{0}(x)
\end{aligned}
$$

to describe the propagation of a weakly nonlinear internal wave propagating at the interface of two immiscible fluids with constant densities $\rho_{1}, \rho_{2}$ with $\rho_{2} / \rho_{1}>1$ (for stable stratification), contained at rest in a long channel with a horizontal rigid top and bottom, and the thickness of the lower layer is assumed to be effectively infinite (deep water limit). The constants $\alpha$ and $\epsilon$ are small positive real numbers that measure the intensity of nonlinear and dispersive effects, respectively. The variable $x$ denotes the spatial position, the variable $t$ is the propagation time, $u=u(x, t)$ represents the velocity monitored at the

* This work was supported by Universidad del Valle, A.A. 25360, Cali, Colombia. 
depth $z=1-\sqrt{2 / 3}$, the function $\zeta=\zeta(x, t)$ is the wave amplitude at the point $x$ and time $t$, measured with respect to the rest level of the two-fluid interface, and $\mathcal{H} f(x)$ denotes the Hilbert transform defined by

$$
\mathcal{H} f(x)=\frac{1}{\pi} \mathrm{p} \cdot \mathrm{v} \cdot \int_{-\infty}^{\infty} \frac{f(y)}{y-x} d y
$$

where p.v. $\int$ stands for the integration in the principal value sense. In the last decades the phenomenon of propagation of waves at the interface between two layers of immiscible fluids has attracted interest of many physicists and mathematicians, for both well-posedness theory, and asymptotic theory due to the challenging modelling, mathematical and numerical difficulties involved in the analysis of this physical system. Some variants of system (1.1) have been derived by Choi and Camassa in $[8,9]$ for accounting different scaling regimes of the problem, where the terms of order $O\left(\epsilon^{2}\right)$ were neglected and $\alpha=O(\epsilon)$. In contrast, we assume that $\alpha=O\left(\epsilon^{2}\right)$. We also point out that following the ideas from $[10,12,13]$, the velocity at an intermediate depth is used as the velocity variable in system (1.1), instead of the depth-average velocity used in previous works. On the other hand, Bona et al. [7] proposed a general method to derive in a systematic way, and for a wide class of scaling regimes, asymptotic models for the propagation of internal waves under the following assumptions: the fluid is ideal, incompressible, irotational, and under the only influence of gravity, the bottom is flat, and the surface tension is negligible. They also proved that these asymptotic systems are consistent with the full Euler equations. An extension of these results to the case of unidimensional non-flat bottoms was presented by Ruiz and Nachbin [17] and in the presence of surface tension by Anh in [2]. Furthermore, he developed in [3] a study of local well-posedness of solutions of some new Boussinesq/Boussinesq and Boussinesq/Full dispersion systems for modelling internal waves derived formally from the Full dispersion/Full dispersion system introduced by Bona et al. [7]. However, we are not aware of some result on regard to the existence and uniqueness of solutions of problem (1.1) and our first purpose in the present paper is to initiate this study. Some higher-order models where terms of order $O\left(\epsilon^{2}\right)$ are not neglected have been derived for instance in [18, p. 283] and in [8, p. 86].

The second goal of this paper is to introduce a numerical scheme that can be used for approximating the solutions of system (1.1). It is important to note that the presence of the nonlocal integral operator $\mathcal{H}$ in system (1.1) makes the analytical and numerical investigation harder than the study of equations with local terms. In this numerical method, the system is discretized in space by the Fourier spectral method and in time by a second-order accurate scheme. The linear terms are treated implicitly in order to improve numerical stability. However, the scheme can be solved as efficiently as an explicit one since the nonlinear terms are treated explicitly. We also establish a result on the convergence of the semidiscrete scheme and the accuracy is checked using some approximate solutions of the system. A potential application of this numerical tool is to explore the range of velocity for which solitary wave solutions of the system exist and to establish if they are orbitally stable/instable under small disturbances. These are interesting problems that are open to our knowledge. 
Some numerical schemes for approximating solutions of scalar unidirectional Benjamin-Ono type equations were proposed for instance by Pelloni and Dougalis [16] and Tomée and Vasudeva [19]. On the other hand, Choi and Camassa [8] solved system (1.1) (without the correction terms $\zeta_{x x t}, u_{x x t}$ ) by using the pseudospectral (collocation) method with periodic boundary conditions in space and a second order leap-frog method for the time integration. The scheme proposed in the present paper uses a Galerkin spectral approach in space for approximating the solutions of system (1.1) which is straightforward to implement in a computer and an implicit-explicit scheme (IMEX) considered in $[1,4]$ for time integration. In contrast, the collocation method used in [8] requires the definition of large differentiation matrices to evaluate nonlinear terms of the form $u u_{x},(\zeta u)_{x}$.

This paper is organized as follows. In Section 2, we derive system (1.1) from the general fluid dynamics equations for the upper and lower fluids. In Section 3 we introduce notation and auxiliary results that will be employed in the analytical results. In Section 4, we study existence, uniqueness and regularity of solutions of system (1.1). Finally in Section 5, we present the numerical solver that can be used for approximating the solutions of the Cauchy problem (1.1).

\section{Governing Equations}

Let us consider a two-fluid system where the densities are $\rho_{i}$ ( $i=1$ for the upper fluid and $i=2$ for the lower fluid), and $\rho_{1}<\rho_{2}$ for stable stratification (see Fig. 1). The fluids are contained at rest in a long channel with a horizontal rigid top and bottom, and the thickness of the lower layer is assumed to be effectively infinite (deep water limit).

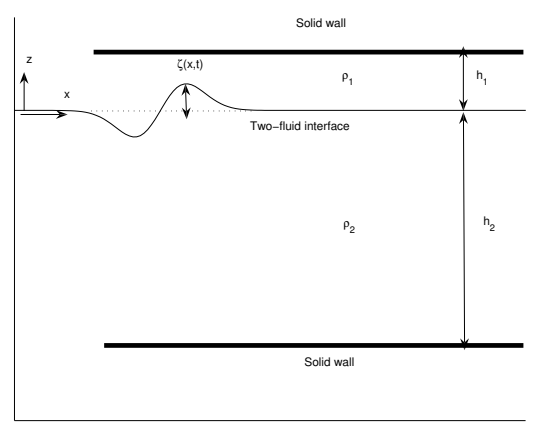

Figure 1. A typical internal wave propagating in a two-fluid system. The rest level of the system is indicated with a dashed line. The system of two layers considered is constrained to a region limited by horizontal rigid lids located at the top and at the bottom.

For inviscid and incompressible fluids, the velocity $\left(u_{i}, w_{i}\right)$ and the pressure 
$p_{i}, i=1,2$ at $(x, z, t)$ satisfy the Euler equations

$$
\begin{aligned}
& u_{i x}+w_{i z}=0, \\
& u_{i t}+u_{i} u_{i x}+w_{i} u_{i z}=-\frac{1}{\rho_{i}} p_{i x}, \\
& w_{i t}+u_{i} w_{i x}+w_{i} w_{i z}=-\frac{1}{\rho_{i}} p_{i z}-g,
\end{aligned}
$$

where $g$ is the gravitational acceleration and subscripts with respect to space and time $x, t$ denote partial differentiation. The boundary conditions at the fluid interface are

$$
\zeta_{t}+u_{1} \zeta_{x}=w_{1}, \quad \zeta_{t}+u_{2} \zeta_{x}=w_{2}, \quad p_{1}=p_{2}
$$

at $z=\zeta(x, t)$. Let $h_{1}, h_{2}>0$ denote the undisturbed thickness of the upper (lower) fluid layer. Then the boundary conditions at the upper and the lower rigid surfaces are

$$
w_{1}\left(x, h_{1}, t\right)=w_{2}\left(x,-h_{2}, t\right)=0, \quad t \geq 0,
$$

respectively.

Let us denote by $\epsilon=h_{1} / L$ (dispersion parameter) and $\alpha=a / h_{1}$ (nonlinear parameter), where the parameters $L, a$ correspond to the characteristic length and amplitude, respectively. Further let $U_{0}=\sqrt{g h_{1}}$ be the characteristic velocity. We are interested in a regime such that $\alpha=O\left(\epsilon^{2}\right)$ with $\alpha, \epsilon$ small.

For irotational fluids, equations (2.1)-(2.5) for the upper fluid layer can be written in terms of the velocity potential $\phi=\phi_{1}$ defined by $\left(\phi_{1 x}, \phi_{1 z}\right)=$ $\left(u_{1}, w_{1}\right)$ :

$$
\begin{aligned}
& \phi_{x x}+\phi_{z z}=0, \quad \zeta(x, t)<z<h_{1}, \\
& \zeta_{t}+\phi_{x} \zeta_{x}=\phi_{z} \quad \text { at } z=\zeta(x, t), \\
& \phi_{t}+\frac{1}{2}\left(\phi_{x}^{2}+\phi_{z}^{2}\right)+g \zeta+\frac{p_{1}}{\rho_{1}}=C(t) \quad \text { at } z=\zeta(x, t), \\
& \phi_{z}\left(x, h_{1}, t\right)=0,
\end{aligned}
$$

where $C(t)$ is a constant that only depends on $t$.

We introduce the scaling

$$
\begin{aligned}
& x=L \tilde{x}, \quad z=h_{1} \tilde{z}, \quad t=\frac{L}{U_{0}} \tilde{t} \\
& \zeta=a \tilde{\zeta}, \quad \phi_{1}=\frac{g L a}{U_{0}} \tilde{\phi}_{1}, \quad p_{1}=\rho_{1} U_{0}^{2} \tilde{p}_{1}
\end{aligned}
$$

to non-dimensionalize all physical variables in equations (2.6)-(2.9). Thus dropping the tildes, the equations in dimensionless variables for the upper layer fluid can be written as 


$$
\begin{aligned}
& \epsilon^{2} \phi_{x x}+\phi_{z z}=0, \quad \alpha \zeta(x, t)<z<1, \\
& \zeta_{t}+\alpha \phi_{x} \zeta_{x}=\frac{1}{\epsilon^{2}} \phi_{z} \quad \text { at } z=\alpha \zeta(x, t), \\
& \phi_{t}+\frac{1}{2}\left(\alpha \phi_{x}^{2}+\frac{\alpha}{\epsilon^{2}} \phi_{z}^{2}\right)+\zeta+\frac{1}{\alpha} p_{1}=C(t) \quad \text { at } z=\alpha \zeta(x, t), \\
& \phi_{z}(x, 1, t)=0 .
\end{aligned}
$$

Let us consider a series expansion around the upper boundary $z=1$, for the velocity potential as in Whitham [20, p. 464]:

$$
\phi(x, z, t)=\sum_{n=0}^{\infty}(1-z)^{n} f_{n}(x, t) .
$$

Substituting in equation (2.11) and using the boundary condition (2.14), we obtain that

$$
\begin{aligned}
\phi(x, z, t) & =\sum_{n=0}^{\infty}\left(-\epsilon^{2}\right)^{n}(1-z)^{2 n} \frac{\partial_{x}^{2 n} f}{(2 n) !} \\
& =f-\epsilon^{2}(1-z)^{2} \frac{f_{x x}}{2}+\epsilon^{4}(1-z)^{4} \frac{f_{x x x x}}{4 !}+O\left(\epsilon^{6}\right),
\end{aligned}
$$

where $f=f_{0}$.

Substituting $\phi(x, z, t)$ in the equations $(2.12)-(2.13)$ for the fluid interface $z=\alpha \zeta(x, t)$, and retaining up to terms of order $O\left(\alpha, \epsilon^{2}\right)$, we obtain the system

$$
\begin{aligned}
& \zeta_{t}-\left((1-\alpha \zeta) f_{x}\right)_{x}+\frac{\epsilon^{2}}{6} \partial_{x}^{4} f=0, \\
& f_{t}-\frac{\epsilon^{2}}{2} f_{x x t}+\frac{1}{2} \alpha f_{x}^{2}+\zeta+\frac{1}{\alpha} p_{1}=C(t) .
\end{aligned}
$$

On the other hand, the equations satisfied by the potential $\phi=\phi_{2}$, and the pressure $p_{2}$ in the lower fluid are given by

$$
\begin{aligned}
& \phi_{x x}+\phi_{z z}=0, \quad-h_{2}<z<\zeta(x, t), \\
& \zeta_{t}+\phi_{x} \zeta_{x}=\phi_{z} \quad \text { at } z=\zeta(x, t), \\
& \phi_{t}+\frac{1}{2}\left(\phi_{x}^{2}+\phi_{z}^{2}\right)+g \zeta+\frac{p_{2}}{\rho_{2}}=C(t) \quad \text { at } z=\zeta(x, t), \\
& \phi_{z}\left(x,-h_{2}, t\right)=0 .
\end{aligned}
$$

For the lower fluid we introduce the following scaling

$$
\begin{aligned}
& x=L \tilde{x}, \quad z=L \tilde{z}, \quad t=\frac{L}{U_{0}} \tilde{t}, \\
& \zeta=a \tilde{\zeta}, \quad \phi_{2}=\frac{\epsilon g L a}{U_{0}} \tilde{\phi}_{2}, \quad p_{2}=\rho_{1} U_{0}^{2} \tilde{p}_{2} .
\end{aligned}
$$

In dimensionless variables and dropping the tildes, the equations for the lower fluid (2.19)-(2.22) transform into 


$$
\begin{aligned}
& \phi_{x x}+\phi_{z z}=0, \quad-h_{2} / L<z<\alpha \epsilon \zeta(x, t), \\
& \zeta_{t}+\alpha \epsilon \phi_{x} \zeta_{x}=\phi_{z}, \quad z=\alpha \epsilon \zeta(x, t), \\
& \alpha \epsilon \phi_{t}+\frac{1}{2} \alpha^{2} \epsilon^{2}\left(\phi_{x}^{2}+\phi_{z}^{2}\right)+\alpha \zeta+\frac{\rho_{1}}{\rho_{2}} P=C(t), \quad z=\alpha \epsilon \zeta(x, t), \\
& \phi_{z}\left(x,-h_{2} / L, t\right)=0,
\end{aligned}
$$

where we define $P(x, t):=p_{1}(x, \alpha \zeta(x, t), t)=p_{2}(x, \alpha \epsilon \zeta(x, t), t)$. Differentiating equation (2.26) with respect to $x$, we obtain the following expression for the pressure $P(x)$ at the fluid interface $z=\alpha \epsilon \zeta(x, t)$ :

$$
P_{x}=-\frac{\rho_{2}}{\rho_{1}} \alpha\left(\zeta_{x}+\epsilon \phi_{x t}\right)+O\left(\alpha^{2} \epsilon^{2}\right) .
$$

Observe now that expanding the potential around $z=0$ we have that

$$
\phi_{x}(x, \alpha \epsilon \zeta(x, t), t)=\phi_{x}(x, 0, t)+O(\alpha \epsilon) .
$$

By virtue of equation (2.25) we have $\phi_{z}=\zeta_{t}+O(\alpha \epsilon)$, at $z=0$. Using the Fourier transform, we solve the Laplace equation (2.24) subject to the Newmantype boundary condition (2.27) to obtain that

$$
\phi_{x}(x, 0, t)=\mathcal{T}\left(\phi_{z}(x, 0, t)\right)=\mathcal{T}\left(\zeta_{t}+O(\alpha \epsilon)\right),
$$

where $\mathcal{T}$ denotes the operator

$$
\mathcal{T}(f)=\frac{1}{2 h} \text { p.v. } \int f\left(x^{\prime}\right) \operatorname{coth}\left(\frac{\pi\left(x^{\prime}-x\right)}{2 h}\right) d x^{\prime}
$$

and $h=h_{2} / L$. Therefore differentiating the equation above with respect to $t$, we have

$$
\phi_{x t}(x, 0, t)=\mathcal{T}\left(\zeta_{t t}+O(\alpha \epsilon)\right) .
$$

Substituting this result in the equation for the pressure (2.28), we get

$$
P_{x}=-\frac{\rho_{2}}{\rho_{1}} \alpha\left(\zeta_{x}+\epsilon \mathcal{T}\left(\zeta_{t t}+O(\alpha \epsilon)\right)\right)+O\left(\alpha^{2} \epsilon^{2}\right) .
$$

From equation (2.17) we arrive at $\zeta_{t}=f_{x x}+O\left(\alpha, \epsilon^{2}\right)$, which implies that

$$
P_{x}=-\frac{\rho_{2}}{\rho_{1}} \alpha\left(\zeta_{x}+\epsilon \mathcal{T}\left(f_{x x t}\right)\right)+O\left(\alpha^{2} \epsilon^{2}\right) .
$$

After differentiating of the momentum equation (2.18) with respect to $x$ and substituting the expression for the pressure given in (2.29), we obtain by neglecting terms of order $O\left(\alpha^{2} \epsilon^{2}\right)$,

$$
f_{x t}-\frac{\epsilon^{2}}{2} \partial_{x}^{3} \partial_{t} f+\frac{1}{2} \alpha\left(f_{x}^{2}\right)_{x}+\zeta_{x}-\frac{\rho_{2}}{\rho_{1}}\left(\zeta_{x}+\epsilon \mathcal{T}\left(\partial_{x}^{2} \partial_{t} f\right)\right)=0 .
$$

We then introduce the velocity $u(x, t)$ monitored at a fixed depth $z=Z_{0}$ with $\alpha \epsilon \zeta<Z_{0}<1$. Since $\zeta$ is order 1 , this inequality is satisfied for $\alpha, \epsilon \ll 1$. 
We point out that this idea was already applied in [13] to obtain a formally equivalent Boussinesq approximation for the problem of water waves propagating over a shallow channel with slowly-variable depth. In $[10,12]$ this idea was applied when the channel's depth is highly varying.

Using equation (2.16), we have that

$$
u(x, t)=\phi_{x}\left(x, Z_{0}, t\right)=f_{x}-\epsilon^{2}\left(1-Z_{0}\right)^{2} \frac{\partial_{x}^{3} f}{2}+\epsilon^{4}\left(1-Z_{0}\right)^{4} \frac{\partial_{x}^{5} f}{4 !}+O\left(\epsilon^{6}\right)
$$

and thus

$$
\begin{aligned}
& f_{x}=u+\frac{\epsilon^{2}}{2}\left(1-Z_{0}\right)^{2} \partial_{x}^{3} f+O\left(\epsilon^{4}\right), \quad f_{x t}=u_{t}+\frac{\epsilon^{2}}{2}\left(1-Z_{0}\right)^{2} \partial_{x}^{3} \partial_{t} f+O\left(\epsilon^{4}\right), \\
& f_{x x}=u_{x}+\frac{\epsilon^{2}}{2}\left(1-Z_{0}\right)^{2} \partial_{x}^{4} f+O\left(\epsilon^{4}\right), \quad \partial_{x}^{4} f=\partial_{x}^{3} u+\frac{\epsilon^{2}}{2}\left(1-Z_{0}\right)^{2} \partial_{x}^{6} f+O\left(\epsilon^{4}\right) .
\end{aligned}
$$

Substituting these expressions in equations (2.17), (2.30) and retaining terms up to order $O\left(\epsilon, \epsilon^{2}, \alpha\right)$, we obtain that

$$
\begin{aligned}
& \zeta_{t}-((1-\alpha \zeta) u)_{x}=\epsilon^{2}\left(\frac{\left(1-Z_{0}\right)^{2}}{2}-\frac{1}{6}\right) \partial_{x}^{3} u \\
& u_{t}+\alpha u u_{x}+\left(1-\frac{\rho_{2}}{\rho_{1}}\right) \zeta_{x}=\frac{\rho_{2}}{\rho_{1}} \epsilon \mathcal{T}\left(u_{x t}\right)+\frac{\epsilon^{2}}{2}\left(1-\left(1-Z_{0}\right)^{2}\right) u_{x x t} .
\end{aligned}
$$

Observe that these equations give that $\zeta_{t}=u_{x}+O\left(\alpha, \epsilon^{2}\right)$, and thus we get the system

$$
\begin{aligned}
& \zeta_{t}-((1-\alpha \zeta) u)_{x}=\epsilon^{2}\left(\frac{\left(1-Z_{0}\right)^{2}}{2}-\frac{1}{6}\right) \zeta_{x x t} \\
& u_{t}+\alpha u u_{x}+\left(1-\frac{\rho_{2}}{\rho_{1}}\right) \zeta_{x}=\frac{\rho_{2}}{\rho_{1}} \epsilon \mathcal{T}\left(u_{x t}\right)+\frac{\epsilon^{2}}{2}\left(1-\left(1-Z_{0}\right)^{2}\right) u_{x x t}
\end{aligned}
$$

In the deep water limit $h=h_{2} / L \rightarrow \infty$, we have that

$$
\lim _{h \rightarrow \infty} \mathcal{T}(f)=\mathcal{H}(f)
$$

and thus the above system transforms into

$$
\begin{aligned}
& \zeta_{t}-((1-\alpha \zeta) u)_{x}=\epsilon^{2}\left(\frac{\left(1-Z_{0}\right)^{2}}{2}-\frac{1}{6}\right) \zeta_{x x t}, \\
& u_{t}+\alpha u u_{x}+\left(1-\frac{\rho_{2}}{\rho_{1}}\right) \zeta_{x}=\frac{\rho_{2}}{\rho_{1}} \epsilon \mathcal{H}\left(u_{x t}\right)+\frac{\epsilon^{2}}{2}\left(1-\left(1-Z_{0}\right)^{2}\right) u_{x x t} .
\end{aligned}
$$

We point out that neglecting the terms of order $O\left(\epsilon^{2}\right)$, we recover a system derived by Choi and Camassa in $[8,9]$. When $Z_{0}=1-\sqrt{2 / 3}$, we obtain system (1.1). Furthermore, for this choice of the parameter $Z_{0}$ the third-order dispersive terms of order $\epsilon^{2}$ become symmetric. In Section 4 , we will see that the presence of these terms enables the use of the Banach fixed point theorem to establish existence and uniqueness of solutions of system (1.1). 


\section{$3 \quad$ Notation and Preliminaries}

In the following results on existence of solutions of system (1.1), we will only require that $1-\rho_{2} / \rho_{1}<0$. All other constants are positive since $\alpha, \epsilon, \rho_{1}, \rho_{2}>0$. Thus that only for simplicity and without loss of generality, we consider system (1.1) with $1-\rho_{2} / \rho_{1}=-1$ and all other coefficients equal to 1 :

$$
\begin{aligned}
& \zeta_{t}-((1-\zeta) u)_{x}=\zeta_{x x t}, \quad x \in \mathbb{R}, t>0 \\
& u_{t}+u u_{x}-\zeta_{x}=\mathcal{H}\left(u_{x t}\right)+u_{x x t}
\end{aligned}
$$

subject to the initial conditions $\zeta(x, 0)=\zeta_{0}(x), u(x, 0)=u_{0}(x)$. To analyze the existence of solutions of system (3.1)-(3.2), we will use the standard notation. For $1 \leq p \leq \infty$ we will denote by $L^{p}(\mathbb{R})$ (or simply $L^{p}$ ) the Banach space of measurable functions in $\mathbb{R}$ such that $\int_{\mathbb{R}}|f(x)|^{p} d x<\infty$ if $1 \leq p<\infty$, and

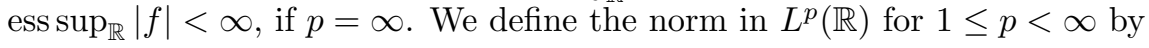

$$
\|f\|_{L^{p}}=\left(\int_{\mathbb{R}}|f(x)|^{p}\right)^{1 / p}
$$

and in $L^{\infty}(\mathbb{R})$ by $\|f\|_{\infty}=\operatorname{ess}_{\sup }|f| . L^{2}(\mathbb{R})$ is a Hilbert space for the scalar product

$$
\langle f, g\rangle=\int_{\mathbb{R}} f(x) \overline{g(x)} d x .
$$

We set $\|f\|=\|f\|_{L^{2}}$. For a function $f \in L^{1}(\mathbb{R})$, the Fourier transform is defined as

$$
\mathcal{F}(f)(y)=\hat{f}(y)=\int_{\mathbb{R}} f(x) e^{-i x y} d x, \quad y \in \mathbb{R}
$$

and the inverse Fourier transform is defined by

$$
\mathcal{F}^{-1}(f)(y)=\check{f}(y)=\frac{1}{2 \pi} \int_{\mathbb{R}} f(x) e^{i x y} d x, \quad y \in \mathbb{R} .
$$

We will also denote by $\mathcal{F}(f)$ (or $\widehat{f}$ ) and $\mathcal{F}^{-1}(f)$ (or $\check{f}$ ) the extensions of these operators to $L^{2}(\mathbb{R})$. The convolution of two functions $f, g \in L^{2}(\mathbb{R})$ is defined as

$$
f * g(x)=\int_{\mathbb{R}} f(x-y) g(y) d y .
$$

We recall that $\widehat{f * g}(y)=\hat{f}(y) \hat{g}(y)$. For $s \in \mathbb{R}$, we define the Sobolev space $H^{s}(\mathbb{R})$ (sometimes written for simplicity $H^{s}$ ) as the completion of the Schwartz space (rapidly-decaying functions) defined as

$$
S(\mathbb{R}):=\left\{f \in C^{\infty}(\mathbb{R}):\left\|x^{\nu} \partial_{x}^{\beta} f\right\|_{\infty}<\infty, \text { for any } \nu, \beta \in \mathbb{Z}^{+}\right\}
$$

with respect to the norm

$$
\|f\|_{H^{s}}=\left(\int_{\mathbb{R}}\left(1+y^{2}\right)^{s}|\hat{f}(y)|^{2} d y\right)^{1 / 2} .
$$


For simplicity, we also denote this norm as $\|f\|_{s}$. The inner product in $H^{s}(\mathbb{R})$ is defined as

$$
\langle f, g\rangle_{s}=\int_{\mathbb{R}}\left(1+y^{2}\right)^{s} \hat{f}(y) \overline{\hat{g}(y)} d y .
$$

The product norm in the space $H^{s}(\mathbb{R}) \times H^{s}(\mathbb{R})$ is defined by

$$
\|(\zeta, u)\|_{H^{s} \times H^{s}}=\|\zeta\|_{H^{s}}+\|u\|_{H^{s}}
$$

for $(\zeta, u) \in H^{s}(\mathbb{R}) \times H^{s}(\mathbb{R})$. For $T>0$, we will denote by $C\left([0, T], H^{s}(\mathbb{R})\right)$ the space of continuous functions $f:[0, T] \rightarrow H^{s}(\mathbb{R})$, i.e. the space of continuous functions $t \rightarrow f(t,.) \in H^{s}(\mathbb{R}), t \in[0, T]$, with the supremum norm

$$
\|f\|_{C(0, T)}=\sup _{t \in[0, T]}\|f(t, .)\|_{H^{s}}
$$

and the product norm

$$
\|(\zeta, u)\|_{C(0, T)^{2}}=\|\zeta\|_{C(0, T)}+\|u\|_{C(0, T)}
$$

for $(\zeta, u) \in C\left([0, T], H^{s}(\mathbb{R}) \times H^{s}(\mathbb{R})\right)$.

An important property of the Hilbert transform which will be used in the sequel is $\widehat{\mathcal{H}(u)}(y)=i \operatorname{sgn}(y) \widehat{u}(y)$, where

$$
\operatorname{sgn}(y)= \begin{cases}|y| / y, & y \neq 0 \\ 0, & y=0\end{cases}
$$

\section{Existence and Uniqueness of Solutions}

In first place, taking the Fourier transform with respect to the variable $x$, system (3.1)-(3.2) can be written as

$$
\begin{aligned}
& \zeta(x, t)=\zeta_{0}(x)+\int_{0}^{t} \mathcal{F}^{-1}\left(\frac{i y}{1+y^{2}}(\hat{u}-\widehat{\zeta u})\right)\left(x, t^{\prime}\right) d t^{\prime}, \\
& u(x, t)=u_{0}(x)+\int_{0}^{t} \mathcal{F}^{-1}\left(\frac{i y}{1+|y|+y^{2}}\left(-\frac{u^{2}}{2}+\hat{\zeta}\right)\right)\left(x, t^{\prime}\right) d t^{\prime} .
\end{aligned}
$$

We will denote the Fourier and the inverse Fourier transforms of the vectorvalued function $f=\left(f_{1}, f_{2}\right)$ as

$$
\mathcal{F}(f)=\left(\mathcal{F} f_{1}, \mathcal{F} f_{2}\right), \quad \mathcal{F}^{-1}(f)=\left(\mathcal{F}^{-1}\left(f_{1}\right), \mathcal{F}^{-1}\left(f_{2}\right)\right),
$$

respectively.

Theorem 1. Let $s>\frac{1}{2}$, and $U_{0}=\left(\zeta_{0}, u_{0}\right)^{T} \in H^{s} \times H^{s}$. Then there exists $T>0$ and a unique $U \in C\left([0, T] ; H^{s} \times H^{s}\right)$, where $U(t)=(\zeta(t, \cdot), u(t, \cdot))^{T}$ which satisfies the integral equation

$$
U(t)=U_{0}+\int_{0}^{t} \mathcal{F}^{-1}\left(F\left(U\left(t^{\prime}\right)\right)\right) d t^{\prime},
$$

where

$$
F(U(t))=\left(\frac{i y}{1+y^{2}}(\hat{u}-\widehat{\zeta u}), \frac{i y}{1+|y|+y^{2}}\left(-\frac{\widehat{u^{2}}}{2}+\hat{\zeta}\right)\right)^{T} .
$$


Proof. Let $M, T>0$, and define the integral operator

$$
\mathcal{A}(U(t))=U_{0}+\int_{0}^{t} \mathcal{F}^{-1}\left(F\left(U\left(t^{\prime}\right)\right)\right) d t^{\prime}
$$

and

$$
X(T)=\left\{U \in C\left([0, T] ; H^{s} \times H^{s}\right):\left\|U(t)-U_{0}\right\|_{C(0, T)^{2}} \leq M\right\} .
$$

It is clear that a fixed point of the operator $\mathcal{A}$ corresponds to a solution of the integral equation (4.1). In first place, we will show that if $U \in X(T)$ then $\mathcal{A}(U) \in X(T)$. Suppose that $\tau, t \in[0, T]$ with $\tau>t \geq 0$. Thus

$$
\begin{aligned}
& \|\mathcal{A}(U(t))-\mathcal{A}(U(\tau))\|_{H^{s} \times H^{s}}=\left\|\int_{t}^{\tau} \mathcal{F}^{-1}\left(F\left(U\left(t^{\prime}\right)\right)\right) d t^{\prime}\right\|_{H^{s} \times H^{s}} \\
& \leq \int_{t}^{\tau}\left(\int_{\mathbb{R}}\left(1+y^{2}\right)^{s}\left|\frac{i y}{1+y^{2}}\right|^{2}|\widehat{u-\zeta u}|^{2} d y\right)^{1 / 2} d t^{\prime} \\
& \quad+\int_{t}^{\tau}\left(\int_{\mathbb{R}}\left(1+y^{2}\right)^{s}\left|\frac{i y}{1+|y|+y^{2}}\right|^{2}\left|-\frac{u^{2}}{2}+\widehat{\zeta}\right|^{2} d y\right)^{1 / 2} d t^{\prime} \\
& \leq|\tau-t| \sup _{t \in[0, T]}\left(\|u(t)-\zeta(t) u(t)\|_{H^{s}}+\left\|\zeta(t)-\frac{u(t)^{2}}{2}\right\|_{H^{s}}\right) .
\end{aligned}
$$

Using the fact that $H^{s}$ is an algebra for $s>1 / 2$, we conclude that $\mathcal{A}(U)$ belongs to $C\left([0, T] ; H^{s} \times H^{s}\right)$.

Let us suppose now that

$$
\sup _{t \in[0, T]}\left\|U(t)-U_{0}\right\|_{H^{s} \times H^{s}} \leq M
$$

Therefore

$$
\|\zeta(t)\|_{H^{s}}+\|u(t)\|_{H^{s}} \leq M+\left\|U_{0}\right\|_{H^{s} \times H^{s}}
$$

for any $t \in[0, T]$. As a consequence,

$$
\begin{aligned}
& \left\|\mathcal{A}(U(t))-U_{0}\right\|_{H^{s} \times H^{s}} \leq\left\|\int_{0}^{t} \mathcal{F}^{-1}\left(F\left(U\left(t^{\prime}\right)\right)\right) d t^{\prime}\right\|_{H^{s} \times H^{s}} \\
& \quad \leq T \sup _{t \in[0, T]}\left(\|u(t)-\zeta(t) u(t)\|_{H^{s}}+\left\|\zeta(t)-\frac{u(t)^{2}}{2}\right\|_{H^{s}}\right) \\
& \quad \leq T \sup _{t \in[0, T]}\left(\|\zeta(t)\|_{H^{s}}\left(1+\|u(t)\|_{H^{s}}\right)+\|u(t)\|_{H^{s}}\left(1+\frac{1}{2}\|u(t)\|_{H^{s}}\right)\right) \\
& \quad \leq T\left(M+\left\|U_{0}\right\|_{H^{s} \times H^{s}}\right)\left(1+M+\left\|U_{0}\right\|_{H^{s} \times H^{s}}\right)<M,
\end{aligned}
$$

provided that $T>0$ is small enough. As a conclusion, we have that if $U \in X(T)$ then $\mathcal{A}(U) \in X(T)$ for a small $T>0$.

In second place, we establish that the operator $\mathcal{A}$ is a contraction from $X(T)$ in itself, provided $T$ is small enough. Let $U=\left(\zeta_{1}, u_{1}\right)^{T}$ and $V=\left(\zeta_{2}, u_{2}\right)^{T}$ be 
in the set $X(T)$. Therefore

$$
\begin{aligned}
&\|\mathcal{A}(U(t))-\mathcal{A}(V(t))\|_{H^{s} \times H^{s}} \leq\left\|\int_{0}^{t} \mathcal{F}^{-1}\left(U\left(t^{\prime}\right)-V\left(t^{\prime}\right)\right) d t^{\prime}\right\|_{H^{s} \times H^{s}} \\
& \leq\left.\int_{0}^{t}\left(\int_{\mathbb{R}}\left(1+y^{2}\right)^{s}\left|\frac{i y}{1+y^{2}}\right|^{2} \mid \widehat{\left(u_{1}\right.}-\widehat{\zeta_{1} u_{1}}\right)-\left.\left(\widehat{u_{2}}-\widehat{\zeta_{2} u_{2}}\right)\right|^{2} d y\right)^{1 / 2} d t^{\prime} \\
&+\int_{0}^{t}\left(\int_{\mathbb{R}}\left(1+y^{2}\right)^{s}\left|\frac{i y}{1+|y|+y^{2}}\right|^{2} \mid\left(-\widehat{u_{1}^{2}}+\widehat{2}+\widehat{\zeta_{1}}\right)-\left(-\frac{\widehat{u_{2}^{2}}}{2}+\widehat{\zeta_{2}}\right) d y\right)^{1 / 2} d t^{\prime} \\
& \leq \int_{0}^{t}\left\|\left(u_{1}\left(t^{\prime}\right)-\zeta_{1}\left(t^{\prime}\right) u_{1}\left(t^{\prime}\right)\right)-\left(u_{2}\left(t^{\prime}\right)-\zeta_{2}\left(t^{\prime}\right) u_{2}\left(t^{\prime}\right)\right)\right\|_{H^{s} \times H^{s}} d t^{\prime} \\
&+\int_{0}^{t}\left\|\left(-\frac{u_{1}\left(t^{\prime}\right)^{2}}{2}+\zeta_{1}\left(t^{\prime}\right)\right)-\left(-\frac{u_{2}\left(t^{\prime}\right)^{2}}{2}+\zeta_{2}\left(t^{\prime}\right)\right)\right\|_{H^{s} \times H^{s}} d t^{\prime} .
\end{aligned}
$$

Therefore

$$
\begin{aligned}
& \|\mathcal{A}(U(t))-\mathcal{A}(V(t))\| \leq T \sup _{t \in[0, T]}\left(\left\|\zeta_{1}-\zeta_{2}\right\|_{H^{s}}+\frac{1}{2}\left(\left\|u_{1}\right\|_{H^{s}}+\left\|u_{2}\right\|_{H^{s}}\right)\right. \\
& \left.\quad \times\left(\left\|u_{1}-u_{2}\right\|_{H^{s}}\right)+\left\|u_{1}-u_{2}\right\|_{H^{s}}+\left\|\zeta_{2}\right\|_{H^{s}}\left\|u_{1}-u_{2}\right\|_{H^{s}}+\left\|u_{1}\right\|_{H^{s}}\left\|\zeta_{1}-\zeta_{2}\right\|_{H^{s}}\right) \\
& \leq T \sup _{t \in[0, T]}\left(\left(1+\left\|u_{1}\right\|_{H^{s}}\right)\left\|\zeta_{1}-\zeta_{2}\right\|_{H^{s}}+\left(\frac{1}{2}\left(\left\|u_{1}\right\|_{H^{s}}+\left\|u_{2}\right\|_{H^{s}}\right)\right.\right. \\
& \left.\left.\quad+1+\left\|\zeta_{2}\right\|_{H^{s}}\right)\left\|u_{1}-u_{2}\right\|_{H^{s}}\right) \\
& \leq T \sup _{t \in[0, T]}\left(\left(1+M+\left\|U_{0}\right\|_{H^{s} \times H^{s}}\right)\left\|\zeta_{1}-\zeta_{2}\right\|_{H^{s}}\right. \\
& \left.\quad+\left(1+2 M+2\left\|U_{0}\right\|_{H^{s} \times H^{s}}\right)\left\|u_{1}-u_{2}\right\|_{H^{s}}\right) \\
& \leq T\left(1+2 M+2\left\|U_{0}\right\|_{H^{s} \times H^{s}}\right) \sup _{t \in[0, T]}\|U(t)-V(t)\|_{H^{s} \times H^{s}} .
\end{aligned}
$$

We see that we can select $\tilde{T} \in(0, T)$ (which depends on the initial condition $U_{0}$ ) such that

$$
\tilde{T}\left(1+2 M+2\left\|U_{0}\right\|_{H^{s} \times H^{s}}\right)<1
$$

we have that the operator is a contraction from $X(\tilde{T})$ in itself. Then the Banach's contraction principle implies the existence of a unique solution in $X(\tilde{T})$ of the integral equation (4.1). The uniqueness of the obtained solution in the space $C\left([0, \tilde{T}] ; H^{s} \times H^{s}\right)$ can be established using Gronwall's lemma. This finishes the proof of the theorem.

Remark 1. As we mention above, the presence of the two dispersive $O\left(\epsilon^{2}\right)$ terms in system (3.1)-(3.2) allowed to use the Banach fixed point theorem to establish local existence and uniqueness of solutions of system (1.1). In contrast, if for instance we set $Z_{0}=1-\sqrt{1 / 3}$, then equations (1.1) reduces to the non- 
regularized system

$$
\begin{aligned}
& \zeta_{t}-((1-\alpha \zeta) u)_{x}=0, \\
& u_{t}+\alpha u u_{x}+\left(1-\frac{\rho_{2}}{\rho_{1}}\right) \zeta_{x}=\frac{\rho_{2}}{\rho_{1}} \epsilon \mathcal{H}\left(u_{x t}\right)+\frac{\epsilon^{2}}{3} u_{x x t}, \\
& \zeta(x, 0)=\zeta_{0}(x), \quad u(x, 0)=u_{0}(x) .
\end{aligned}
$$

Observe that equation (4.3) does not have the dispersive term of order $O\left(\epsilon^{2}\right)$. This has an effect on the smoothness properties of the corresponding nonlinear operator $\mathcal{A}$. We can not use directly the Banach fixed point principle to study existence of solutions of this system, because the operator $\mathcal{A}$ does not map the ball $X(T) \subset H^{s}$ in $X(T)$. This is also the case of previous models derived in [8] and [18] where a kinematic equation in the form of equation (4.3) was employed. Furthermore, the new formulation (2.31)-(2.32) was stated in terms of the fluid velocity at a fixed depth $Z_{0}$, which is easier of measuring than the commonly used depth-averaged velocity employed for instance in $[8,9]$. Regarding possible engineering applications, this is an important advantage of the family of systems (2.31)-(2.32). Other important property of the particular formulation (1.1) is the fact that solutions at time $t$ can be bounded in terms of the initial data, which enables the use of classical tools to extend these solutions for any time $t>0$. This will be shown in the following lemma.

Lemma 1. Let $s>1 / 2$ and $\zeta, u \in C\left([0, T], H^{s}\right)$ be the solution of problem (3.1)-(3.2). Then

$$
\|\zeta(t)\|_{s}^{2}+\|u(t)\|_{s}^{2} \leq C\left(\left\|\zeta_{0}\right\|_{s}^{2}+\left\|\zeta_{0 x}\right\|_{s}^{2}+\left\|u_{0 x}\right\|_{s}^{2}+\left\|u_{0}\right\|_{X^{s}}^{2}\right)
$$

for any $0 \leq t \leq T$, where

$$
\|u\|_{X^{s}}^{2}:=\int_{\mathbb{R}}\left(1+y^{2}\right)^{s}(1+|y|)|\hat{u}(y)|^{2} d y .
$$

Proof. Multiplying equation (3.1) by $\zeta$, equation (3.2) by $u$, and using integration by parts, we obtain

$$
\begin{aligned}
& \left\langle\zeta_{t}, \zeta\right\rangle_{s}+\left\langle(1-\zeta) u, \zeta_{x}\right\rangle_{s}=-\left\langle\zeta_{x t}, \zeta_{x}\right\rangle_{s}, \\
& \left\langle u, u_{t}\right\rangle_{s}-\left\langle u_{x}, \frac{u^{2}}{2}\right\rangle_{s}+\left\langle u_{x}, \zeta\right\rangle_{s}=-\left\langle u_{x}, \mathcal{H}\left(u_{t}\right)\right\rangle_{s}-\left\langle u_{x}, u_{x t}\right\rangle_{s} .
\end{aligned}
$$

Therefore adding the equations above, we find

$$
\frac{1}{2} \partial_{t}\|\zeta\|_{s}^{2}+\frac{1}{2} \partial_{t}\left\|\zeta_{x}\right\|_{s}^{2}+\frac{1}{2} \partial_{t}\|u\|_{s}^{2}+\frac{1}{2} \partial_{t}\left\|u_{x}\right\|_{s}^{2}+\left\langle u_{x}, \mathcal{H}\left(u_{t}\right)\right\rangle_{s}=\left\langle\zeta u, \zeta_{x}\right\rangle_{s}+\left\langle u_{x}, \frac{u^{2}}{2}\right\rangle_{s} .
$$

Thus using the Cauchy- Schwartz inequality and the embedding $H^{s} \subset L^{\infty}$, we get for $0 \leq t \leq T$,

$$
\begin{aligned}
\partial_{t}\left(\|\zeta\|_{s}^{2}+\left\|\zeta_{x}\right\|_{s}^{2}+\left\|u_{x}\right\|_{s}^{2}+\|u\|_{X^{s}}^{2}\right) & \leq 2\left(\|\zeta\|_{\infty}\|u\|_{s}\left\|\zeta_{x}\right\|_{s}+\|u\|_{\infty}\|u\|_{s}\left\|u_{x}\right\|_{s}\right) \\
& \leq C\left(\|\zeta\|_{s}^{2}+\left\|\zeta_{x}\right\|_{s}^{2}+\left\|u_{x}\right\|_{s}^{2}+\|u\|_{X^{s}}^{2}\right) .
\end{aligned}
$$

The result follows as a consequence of the Gronwall's lemma. 
Remark 2. As a consequence of Lemma 1, we have that the local solutions of the integral equation (4.1) guaranteed by Theorem 1 can be extended to any interval $[0, T]$.

\section{Numerical Results}

In this section we will describe a numerical scheme which can be used for approximating the solution of system $(2.31)-(2.32)$ with $Z_{0}=1-\sqrt{2 / 3}$ :

$$
\begin{aligned}
& \zeta_{t}-((1-\alpha \zeta) u)_{x}=\epsilon^{2} \zeta_{x x t} / 6, \\
& u_{t}+\alpha u u_{x}+\left(1-\rho_{r}\right) \zeta_{x}=\rho_{r} \epsilon \mathcal{H}\left(u_{x t}\right)+\epsilon^{2} u_{x x t} / 6, \\
& \zeta(x, 0)=\zeta_{0}(x), \quad u(x, 0)=u_{0}(x)
\end{aligned}
$$

where $\rho_{r}=\rho_{2} / \rho_{1}$. To proceed, the spatial computational domain $[0, L]$ is discretized by $N \in 2 \mathbb{Z}$ equidistant points, with spacing $\Delta x=L / N$. Then, we approximate the unknowns $u$ and $\zeta$ as truncated Fourier series in space with time-dependent coefficients:

$$
u(x, t)=\sum_{j} \widehat{u}_{j}(t) e^{i w_{j} x}, \quad \zeta(x, t)=\sum_{j} \hat{\zeta}_{j}(t) e^{i w_{j} x}
$$

with $w_{j}=2 \pi j / L, j=-N / 2+1, \ldots 0, \ldots N / 2$. The time-dependent coefficients $\widehat{u}_{j}(t)$ for $j=-N / 2+1, \ldots 0, \ldots N / 2$ are calculated by means of the equation

$$
\widehat{u}_{j}(t)=\frac{1}{L} \int_{0}^{L} u(x, t) e^{-i w_{j} x} d x,
$$

and similarly for $\hat{\zeta}_{j}(t)$. Actually, this Fourier strategy allows us to seek approximations of solutions to system (5.1) on a periodic domain $[0, L]$. However, since in this paper we are mainly interested in solutions on $(-\infty, \infty)$ which decay rapidly to zero when $|x| \rightarrow \infty$, such as solitary waves and Gaussian-type initial data, we can take the length of the computational domain $L>0$ large enough in order to the solution does not reach the computational boundaries $x=0$, $x=L$. Thus we are able of computing the time evolution of solutions in these non-periodic problems.

Projecting equations (5.1) with respect to the orthonormal basis $\phi_{j}=$ $L^{-1 / 2} e^{i w_{j} x}$ and the inner product

$$
\langle f, g\rangle=\int_{0}^{L} f(x) \overline{g(x)} d x
$$

we derive that

$$
\begin{aligned}
& \left\langle\zeta_{t}, \phi_{j}\right\rangle-\left\langle(u-\alpha \zeta u)_{x}, \phi_{j}\right\rangle=\frac{\epsilon^{2}}{6}\left\langle\zeta_{x x t}, \phi_{j}\right\rangle \\
& \left\langle u_{t}, \phi_{j}\right\rangle+\frac{\alpha}{2}\left\langle\left(u^{2}\right)_{x}, \phi_{j}\right\rangle+\left(1-\rho_{r}\right)\left\langle\zeta_{x}, \phi_{j}\right\rangle=\rho_{r} \epsilon\left\langle\mathcal{H}\left(u_{x t}\right), \phi_{j}\right\rangle+\frac{\epsilon^{2}}{6}\left\langle u_{x x t}, \phi_{j}\right\rangle .
\end{aligned}
$$


Now substituting the Fourier expansions (5.2) into equations (5.3) and using the orthogonal property of the basis $\phi_{j}$ and integration by parts, we obtain

$$
\begin{aligned}
& \hat{\zeta}_{j}^{\prime}(t)-i w_{j} P_{j}\left[(u-\alpha \zeta u]=-\frac{\epsilon^{2}}{6} w_{j}^{2} \hat{\zeta}_{j}^{\prime},\right. \\
& \hat{u}_{j}^{\prime}(t)+\frac{i \alpha w_{j}}{2} P_{j}\left[u^{2}\right]+i w_{j}\left(1-\rho_{r}\right) \hat{\zeta}_{j}=\rho_{r} \epsilon \sum_{s} i w_{s} \widehat{u}_{s}^{\prime}(t)\left\langle\mathcal{H}\left(\phi_{s}\right), \phi_{j}\right\rangle-\frac{\epsilon^{2}}{6} w_{j}^{2} \hat{u}_{j}^{\prime},
\end{aligned}
$$

where $P_{j}[$.$] is the operator defined by$

$$
P_{j}[g]=\frac{1}{L} \int_{0}^{L} g(x) e^{-i w_{j} x} d x .
$$

Then using the properties of the Hilbert transform, system (5.4) reduces into

$$
\begin{aligned}
& \hat{\zeta}_{j}^{\prime}(t)-i w_{j} P_{j}\left[(u-\alpha \zeta u]=-\frac{\epsilon^{2}}{6} w_{j}^{2} \hat{\zeta}_{j}^{\prime},\right. \\
& \widehat{u}_{j}^{\prime}(t)+\frac{i \alpha w_{j}}{2} P_{j}\left[u^{2}\right]+i w_{j}\left(1-\rho_{r}\right) \hat{\zeta}_{j}=-\rho_{r} \epsilon\left|w_{j}\right| \widehat{u}_{j}^{\prime}-\frac{\epsilon^{2}}{6} w_{j}^{2} \hat{u}_{j}^{\prime} .
\end{aligned}
$$

Finally, we reach expressions for the Fourier coefficients of the unknowns $u$ and $\zeta$ :

$$
\begin{aligned}
& \hat{\zeta}_{j}^{\prime}=\frac{i w_{j}}{1+\frac{\epsilon^{2}}{6} w_{j}^{2}} P_{j}[(u-\alpha \zeta u)], \\
& \widehat{u}_{j}^{\prime}=\frac{i w_{j}\left(\rho_{r}-1\right) \hat{\zeta}_{j}}{1+\rho_{r} \epsilon\left|w_{j}\right|+\frac{\epsilon^{2}}{6} w_{j}^{2}}-\frac{i \alpha w_{j} P_{j}\left[u^{2}\right]}{2\left(1+\rho_{r} \epsilon\left|w_{j}\right|+\frac{\epsilon^{2}}{6} w_{j}^{2}\right)},
\end{aligned}
$$

subject to $\hat{\zeta}_{j}(0)=\hat{\zeta}_{0 j}, \hat{u}_{j}(0)=\hat{u}_{0 j}$. Equations (5.6) can be considered as a system of ordinary differential equations for each frequency $w_{j}$, which we discretized numerically by the following second-order scheme proposed in $[1,4]$ :

$$
\begin{aligned}
\frac{\hat{\zeta}_{j}^{(n+1)}-\hat{\zeta}_{j}^{(n)}}{\Delta t}= & \frac{i w_{j}}{2}\left(\frac{\hat{u}_{j}^{(n+1)}+\hat{u}_{j}^{(n)}}{1+\frac{\epsilon^{2}}{6} w_{j}^{2}}\right)-\frac{3}{2}\left(\frac{i w_{j}}{1+\frac{\epsilon^{2}}{6} w_{j}^{2}}\right) P_{j}[\alpha \zeta u]^{(n)} \\
& +\frac{1}{2}\left(\frac{i w_{j}}{1+\frac{\epsilon^{2}}{6} w_{j}^{2}}\right) P_{j}[\alpha \zeta u]^{(n-1)} \\
\frac{\widehat{u}_{j}^{(n+1)}-\widehat{u}_{j}^{(n)}}{\Delta t}= & \frac{i w_{j}\left(\rho_{r}-1\right)\left(\hat{\zeta}_{j}^{(n+1)}+\hat{\zeta}_{j}^{(n)}\right)}{2\left(1+\rho_{r} \epsilon\left|w_{j}\right|+\frac{\epsilon^{2}}{6} w_{j}^{2}\right)}-\frac{3 i \alpha w_{j} P_{j}\left[u^{2}\right]^{(n)}}{4\left(1+\rho_{r} \epsilon\left|w_{j}\right|+\frac{\epsilon^{2}}{6} w_{j}^{2}\right)} \\
& +\frac{i \alpha w_{j} P_{j}\left[u^{2}\right]^{(n-1)}}{4\left(1+\rho_{r} \epsilon\left|w_{j}\right|+\frac{\epsilon^{2}}{6} w_{j}^{2}\right)} .
\end{aligned}
$$

Here $\Delta t$ denotes the time step and $\widehat{u}_{j}^{(n)}, \hat{\zeta}_{j}^{(n)}$ denote the numerical approximations of the Fourier coefficients $\widehat{u}_{j}(t), \hat{\zeta}_{j}(t)$, respectively, at time $t=n \Delta t$. Also the notation $P_{j}[g]^{(n)}$ means the value of $P_{j}[g]$ when $g$ is evaluated at time $t=n \Delta t$. This implicit-explicit (IMEX) scheme is a combination of a secondorder Adams-Bashforth for the linear terms and a Crank-Nicholson for the nonlinear terms. The main idea behind the IMEX schemes is to integrate implicitly 
the linear terms for obtaining schemes with weak stability time-step restrictions. The convective terms are treated explicitly for ease of implementation. Observe that scheme (5.7) deals with three levels in the temporal evolution. To initiate the scheme we need the approximations of the pair solution $(\zeta, u)$ at two different levels of time. It is clear that the first level is given by the initial conditions $\zeta_{0}(x), u_{0}(x)$. To compute the additional temporal level we may use, for instance, a single-step method to integrate equations (5.6). An experimental analysis of several IMEX schemes, including the one used in (5.7), was carried out in [4] and [5]. Recently, the author also used this IMEX scheme to approximate the solutions of a weakly-nonlinear, weakly-dispersive Boussinesqtype system with highly-variable coefficients [11]. Furthermore, convergence of the fully-discrete numerical solver was analyzed in that work.

\subsection{Linear stability analysis}

Let us consider the linear version of the solver (5.7) (i.e. with $\alpha=0$ ):

$$
\begin{aligned}
& \hat{\zeta}_{j}^{(n+1)}=\hat{\zeta}_{j}^{(n)}+\alpha_{1}\left(\hat{u}_{j}^{(n+1)}+\hat{u}_{j}^{(n)}\right), \\
& \hat{u}_{j}^{(n+1)}=\beta_{1}\left(\hat{\zeta}_{j}^{(n+1)}+\hat{\zeta}_{j}^{(n)}\right)+\hat{u}_{j}^{(n)},
\end{aligned}
$$

where

$$
\alpha_{1}=\frac{i w_{j} \Delta t}{1+\frac{\epsilon^{2}}{6} w_{j}^{2}}, \quad \beta_{1}=\frac{i w_{j}\left(\rho_{r}-1\right) \Delta t}{2\left(1+\rho_{r} \epsilon\left|w_{j}\right|+\frac{\epsilon^{2}}{6} w_{j}^{2}\right)} .
$$

Therefore

$$
\left(\begin{array}{l}
\hat{\zeta}_{j}^{(n+1)} \\
\hat{u}_{j}^{(n+1)}
\end{array}\right)=\frac{1}{1-\alpha_{1} \beta_{1}}\left(\begin{array}{cc}
1+\alpha_{1} \beta_{1} & 2 \alpha_{1} \\
2 \beta_{1} & 1+\alpha_{1} \beta_{1}
\end{array}\right)\left(\begin{array}{l}
\hat{\zeta}_{j}^{(n)} \\
\hat{u}_{j}^{(n)}
\end{array}\right) .
$$

Since $\alpha_{1} \beta_{1}<0$, we get that the eigenvalues of the matrix above are

$$
\lambda_{ \pm}=\frac{1+\alpha_{1} \beta_{1} \pm 2 i \sqrt{-\alpha_{1} \beta_{1}}}{1-\alpha_{1} \beta_{1}} .
$$

Thus $\left|\lambda_{ \pm}\right|=1$, which implies that the linear numerical scheme (5.8) is unconditionally stable (i.e. no upper limit on time step $\Delta t$ ).

\subsection{Error analysis of the semidiscrete scheme for the periodic prob- lem}

We will analyze the convergence of the numerical solver described above applied to the system

$$
\begin{aligned}
& \zeta_{t}-((1-\zeta) u)_{x}=\zeta_{x x t}, \\
& u_{t}+u u_{x}-\zeta_{x}=\mathcal{H}\left(u_{x t}\right)+u_{x x t},
\end{aligned}
$$

subject to the initial conditions $\zeta(x, 0)=\zeta_{0}(x), u(x, 0)=u_{0}(x)$, and $\zeta, u$ and their derivatives are $L$-periodic with respect to the spatial variable $x$.

We set

$$
L^{2}(0, L):=\left\{f:(0, L) \rightarrow \mathbb{C},\|f\|=\left[\int_{0}^{L}|f(x)|^{2} d x\right]^{1 / 2}<\infty\right\}
$$


with the inner product $\langle f, g\rangle=\int_{0}^{L} f(x) \overline{g(x)} d x$, and let $C_{\#}^{\infty}(0, L)$ be the space of all infinitely differentiable functions that are $L$-periodic so as all their derivatives. Moreover, for any integer $r \geq 0, H_{\#}^{r}(0, L)$ stands for the closure of $C_{\#}^{\infty}(0, L)$ in the Sobolev space

$$
H^{r}(0, L):=\left\{f \in L^{2}(0, L),\|f\|_{r}=\left[\sum_{j=0}^{r}\left\|\partial^{j} f / \partial x^{j}\right\|^{2}\right]^{1 / 2}<\infty\right\} .
$$

Let $S_{N}$ be the finite dimensional space generated by $\phi_{j}=L^{-1 / 2} e^{\frac{2 \pi}{T} j x}, j=$ $-N / 2, \ldots N / 2, N \in 2 \mathbb{Z}$ and $P_{N}: L^{2}(0, L) \rightarrow S_{N}$ be the orthogonal projection on the space $S_{N}$,

$$
P_{N} g:=\sum_{j=-N / 2+1}^{N / 2} \hat{g}_{j} \phi_{j}
$$

with $\hat{g}_{j}=\int_{0}^{L} g(x) \overline{\phi_{j}(x)} d x$. For any $\phi \in S_{N}$, and $g \in L^{2}(0, L)$, we have that

$$
\int_{0}^{L}\left(P_{N} g-g\right) \bar{\phi} d x=0 .
$$

Furthermore, for all $g \in L^{2}(0, L),\left(P_{N} g\right)_{N \in 2 \mathbb{N}}$ converges to $g$ and for any integers $r \geq s, s \geq 0$ one has (see [15]) that

$$
\left\|P_{N} g-g\right\|_{s} \leq C N^{s-r}\|g\|_{r}
$$

for all $g \in H_{\#}^{r}(0, L)$. For $T>0$ the weak formulation of problem (5.9) is to find $\zeta, u \in C\left([0, T], H_{\#}^{1}(0, L)\right)$ such that

$$
\begin{aligned}
& \left\langle\zeta_{t}, \phi\right\rangle+\left\langle(1-\zeta) u, \phi^{\prime}\right\rangle=-\left\langle\zeta_{x t}, \phi^{\prime}\right\rangle, \\
& \left\langle u_{t}, \psi\right\rangle-\left\langle\frac{u^{2}}{2}, \psi^{\prime}\right\rangle+\left\langle\zeta, \psi^{\prime}\right\rangle=-\left\langle\mathcal{H}\left(u_{t}\right), \psi^{\prime}\right\rangle-\left\langle u_{x t}, \psi^{\prime}\right\rangle, \\
& \zeta(0)=\zeta_{0}, \quad u(0)=u_{0}
\end{aligned}
$$

for all $\phi, \psi \in H_{\#}^{1}(0, L), 0 \leq t \leq T$.

The semidiscrete Fourier-Galerkin spectral scheme to solve problem (5.11) is to find $\zeta_{N}, u_{N} \in C\left([0, T], S_{N}\right)$ such that

$$
\begin{aligned}
& \left\langle\zeta_{N t}, \phi\right\rangle+\left\langle\left(1-\zeta_{N}\right) u_{N}, \phi^{\prime}\right\rangle=-\left\langle\zeta_{N x t}, \phi^{\prime}\right\rangle, \\
& \left\langle u_{N t}, \psi\right\rangle-\left\langle\frac{u_{N}^{2}}{2}, \psi^{\prime}\right\rangle+\left\langle\zeta_{N}, \psi^{\prime}\right\rangle=-\left\langle\mathcal{H}\left(u_{N t}\right), \psi^{\prime}\right\rangle-\left\langle u_{N x t}, \psi^{\prime}\right\rangle, \\
& \zeta_{N}(0)=P_{N}\left(\zeta_{0}\right), \quad u_{N}(0)=P_{N}\left(u_{0}\right)
\end{aligned}
$$

for all $\phi, \psi \in S_{N}, 0 \leq t \leq T$.

Theorem 2. Let $\zeta, u \in C\left([0, T], H_{\#}^{r}(0, L)\right)$ be the solution of problem (5.11), with $r \geq 2$ integer. Then the semidiscrete problem (5.12) has a unique solution $\zeta_{N}, u_{N} \in C\left([0, T], S_{N}\right)$ and for $N$ sufficiently large there exists a constant $C>0$, independent of $N, t$, such that

$$
\left\|\zeta(t)-\zeta_{N}\right\|+\left\|u(t)-u_{N}(t)\right\| \leq C N^{1-r}
$$

for any $0 \leq t \leq T$. 
Proof. The first observation is that problem (5.12) is equivalent to the system (5.6). From the classical theory of existence of solutions of ordinary differential equations, this system has a unique local solution $\zeta_{N}, u_{N} \in C\left([0, \tilde{T}], S_{N}\right)$ for some $\tilde{T}>0$. This local solution can be extended to any interval $[0, T]$ due to the estimate

$$
\left\|\zeta_{N}(t)\right\|^{2}+\left\|u_{N}(t)\right\|^{2} \leq C\left(\left\|\zeta_{N}(0)\right\|^{2}+\left\|\zeta_{N x}(0)\right\|^{2}+\left\|u_{N x}(0)\right\|^{2}+\left\|u_{N}(0)\right\|_{X^{0}}^{2}\right)
$$

for $0 \leq t \leq T$, which can be easily derived from equations (5.12), using a similar technique as in Lemma 1. Here we remark the importance of the dispersive terms of order $O\left(\epsilon^{2}\right)$ for the validity of the previous inequality, which means that the solution of the semidiscrete formulation at time $t$ can be bounded in terms of the initial data.

Here

$$
\|u\|_{X^{0}}^{2}:=\int_{0}^{L}(1+|y|)|\hat{u}(y)|^{2} d y .
$$

Let

$$
\theta:=P_{N} \zeta-\zeta_{N}, \quad \xi:=P_{N} u-u_{N}, \quad \rho:=\zeta-P_{N} \zeta, \quad \sigma:=u-P_{N} u .
$$

Using the properties of the orthogonal projection we have that

$$
\begin{aligned}
\left\langle P_{N} \zeta_{t}-P_{N} u_{x}+P_{N}\left((\zeta u)_{x}\right), \phi\right\rangle & =\left\langle P_{N}\left(\zeta_{x x t}\right), \phi\right\rangle, \\
\left\langle\psi, P_{N} u_{t}+P_{N}\left(u u_{x}\right)-P_{N}\left(\zeta_{x}\right)\right\rangle & =\left\langle\psi, \mathcal{H}\left(P_{N}\left(u_{x t}\right)\right)+P_{N}\left(u_{x x t}\right)\right\rangle
\end{aligned}
$$

for any $\phi, \psi \in S_{N}$. Observe that the semidiscrete formulation (5.12) can also be written as

$$
\begin{aligned}
& \left\langle\zeta_{N t}-u_{N x}+\left(\zeta_{N} u_{N}\right)_{x}, \phi\right\rangle=\left\langle\zeta_{N x x t}, \phi\right\rangle, \\
& \left\langle\psi, u_{N t}+u_{N} u_{N x}-\zeta_{N x}\right\rangle=\left\langle\psi, \mathcal{H}\left(u_{N x t}\right)+u_{N x x t}\right\rangle .
\end{aligned}
$$

Subtracting equations (5.13)-(5.15) and (5.14)-(5.16), we have that

$$
\begin{aligned}
& \left\langle\partial_{t}\left(P_{N} \zeta-\zeta_{N}\right), \phi\right\rangle-\left\langle P_{N} u_{x}-u_{N x}, \phi\right\rangle+\left\langle P_{N}\left((\zeta u)_{x}\right)-\left(\zeta_{N} u_{N}\right)_{x}, \phi\right\rangle \\
& \quad=\left\langle\partial_{t}\left(P_{N}\left(\zeta_{x x}\right)-\zeta_{N x x}\right), \phi\right\rangle, \\
& \left\langle\psi, \partial_{t}\left(P_{N} u-u_{N}\right)\right\rangle+\left\langle\psi, P_{N}\left(u u_{x}\right)-u_{N} u_{N x}\right\rangle-\left\langle\psi, P_{N} \zeta_{x}-\zeta_{N x}\right\rangle \\
& \quad=\left\langle\psi, \mathcal{H}\left(P_{N}\left(u_{x t}\right)-u_{N x t}\right)\right\rangle+\left\langle\psi, P_{N}\left(u_{x x t}\right)-u_{N x x t}\right\rangle .
\end{aligned}
$$

Suppose that there exists a maximal temporal instance $0<T_{N}<T$ such that

$$
\|\theta(t)\|_{\infty} \leq 1
$$

for $0 \leq t \leq T_{N}$. Furthermore, since $u, \zeta \in C\left([0, T], H_{\#}^{r}(0, L)\right), r \geq 2$, we know that $\|u(t)\|_{1} \leq M,\|\zeta(t)\|_{1} \leq M, 0 \leq t \leq T$, for some constant $M>0$.

We recall that $\theta, \xi$ belong to $S_{N}$. Thus we can let $\phi=\theta$ and $\psi=\xi$ in the equations above to find that

$$
\begin{aligned}
& \left\langle\theta_{t}, \theta\right\rangle-\left\langle\xi_{x}, \theta\right\rangle+\left\langle P_{N}\left((\zeta u)_{x}\right)-\left(\zeta_{N} u_{N}\right)_{x}, \theta\right\rangle=\left\langle\theta_{x x t}, \theta\right\rangle \\
& \left\langle\xi, \xi_{t}\right\rangle+\left\langle\xi, P_{N}\left(u u_{x}\right)-u_{N} u_{N x}\right\rangle-\left\langle\xi, \theta_{x}\right\rangle=\left\langle\xi, \mathcal{H} \xi_{x t}\right\rangle+\left\langle\xi, \xi_{x x t}\right\rangle .
\end{aligned}
$$


Adding these equations and using integration by parts, we obtain that

$$
\begin{aligned}
& \frac{1}{2} \partial_{t}\|\theta\|^{2}+\frac{1}{2} \partial_{t}\left\|\theta_{x}\right\|^{2}+\frac{1}{2} \partial_{t}\|\xi\|_{X^{0}}^{2}+\frac{1}{2} \partial_{t}\left\|\xi_{x}\right\|^{2} \\
& \quad=-\left\langle P_{N}\left((\zeta u)_{x}\right)-\left(\zeta_{N} u_{N}\right)_{x}, \theta\right\rangle-\left\langle\xi, P_{N}\left(u u_{x}\right)-u_{N} u_{N x}\right\rangle
\end{aligned}
$$

In order to bound the right side terms of equality (5.20), observe that

$$
\begin{aligned}
-\langle & \left.P_{N}\left((\zeta u)_{x}\right)-\left(\zeta_{N} u_{N}\right)_{x}, \theta\right\rangle=-\left\langle(\zeta u)_{x}-\left(\zeta_{N} u_{N}\right)_{x}, \theta\right\rangle=\left\langle\zeta u-\zeta_{N} u_{N}, \theta_{x}\right\rangle \\
= & \left\langle u(\rho+\theta)-(\rho+\theta)(\sigma+\xi)+\zeta(\sigma+\xi), \theta_{x}\right\rangle=\left\langle u \rho, \theta_{x}\right\rangle+\left\langle u \theta, \theta_{x}\right\rangle \\
& -\left\langle\rho \sigma, \theta_{x}\right\rangle-\left\langle\rho \xi, \theta_{x}\right\rangle-\left\langle\theta \sigma, \theta_{x}\right\rangle-\left\langle\theta \xi, \theta_{x}\right\rangle+\left\langle\zeta \sigma, \theta_{x}\right\rangle+\left\langle\zeta \xi, \theta_{x}\right\rangle \\
\leq & \|u\|_{\infty}\|\rho\|\left\|\theta_{x}\right\|+\|u\|_{\infty}\|\theta\|\left\|\theta_{x}\right\|+\|\rho\|_{\infty}\|\sigma\|\left\|\theta_{x}\right\|+\|\rho\|_{\infty}\|\xi\|\left\|\theta_{x}\right\| \\
& +\|\sigma\|_{\infty}\|\theta\|\left\|\theta_{x}\right\|+\|\theta\|_{\infty}\|\xi\|\left\|\theta_{x}\right\|+\|\sigma\|_{\infty}\|\zeta\|\left\|\theta_{x}\right\|+\|\zeta\|_{\infty}\|\xi\|\left\|\theta_{x}\right\|,
\end{aligned}
$$

and furthermore,

$$
\begin{aligned}
\left\langle\xi, u_{N} u_{N x}-P_{N}\left(u u_{x}\right)\right\rangle= & \left\langle\xi, u_{N} u_{N x}-u u_{x}\right\rangle \\
= & \left\langle\xi, \xi \xi_{x}+\sigma \sigma_{x}+(\sigma \xi)_{x}-(u \sigma)_{x}-(u \xi)_{x}\right\rangle \\
= & \left\langle\xi, \sigma \sigma_{x}\right\rangle+\left\langle\xi,(\sigma \xi)_{x}\right\rangle-\left\langle\xi,(u \sigma)_{x}\right\rangle-\left\langle\xi,(u \xi)_{x}\right\rangle \\
\leq & \|\sigma\|_{\infty}\left\|\sigma_{x}\right\|\|\xi\|+\left\|\sigma_{x}\right\|_{\infty}\|\xi\|^{2}+\left\|u_{x}\right\|_{\infty}\|\xi\|\|\sigma\| \\
& +\|u\|_{\infty}\left\|\sigma_{x}\right\|\|\xi\|+\left\|u_{x}\right\|_{\infty}\|\xi\|^{2} .
\end{aligned}
$$

Using (5.10), we have that

$$
\begin{aligned}
& \|\rho\| \leq\|\rho\|_{1} \leq C N^{1-r}\|\zeta\|_{r} \leq C, \\
& \|\sigma\| \leq\|\sigma\|_{1} \leq C N^{1-r}\|u\|_{r} \leq C,
\end{aligned}
$$

where $C$ is a constant independent of $N$. Furthermore, we know that due to the embedding $H_{\#}^{1} \subset L^{\infty}$, we have that $\|\rho\|_{\infty} \leq C\|\rho\|_{1},\|\sigma\|_{\infty} \leq C\|\sigma\|_{1}$. Thus from inequality (5.20) and (5.17), we get that

$$
\begin{aligned}
& \frac{1}{2} \partial_{t}\|\theta\|^{2}+\frac{1}{2} \partial_{t}\left\|\theta_{x}\right\|^{2}+\frac{1}{2} \partial_{t}\|\xi\|_{X^{0}}^{2}+\frac{1}{2} \partial_{t}\left\|\xi_{x}\right\|^{2} \\
& \quad \leq C\left(\|\rho\|^{2}+\left\|\theta_{x}\right\|^{2}+\|\theta\|^{2}+\|\sigma\|^{2}+\|\xi\|^{2}+\|\sigma\|_{\infty}^{2}+\|\xi\|_{X^{0}}^{2}+\left\|\sigma_{x}\right\|^{2}\right) .
\end{aligned}
$$

Therefore by virtue of (5.21), we obtain that

$$
\begin{aligned}
& \partial_{t}\left(\|\theta\|^{2}+\left\|\theta_{x}\right\|^{2}+\|\xi\|_{X^{0}}^{2}+\left\|\xi_{x}\right\|^{2}\right) \\
& \quad \leq C\left(\|\theta\|^{2}+\left\|\theta_{x}\right\|^{2}+\|\xi\|_{X^{0}}^{2}+\left\|\xi_{x}\right\|^{2}\right)+C N^{2-2 r}
\end{aligned}
$$

for $0 \leq t \leq T_{N}$, where $C$ is a constant independent of $N$. Using Gronwall's lemma, we obtain that

$$
\|\theta(t)\|^{2}+\left\|\theta_{x}(t)\right\|^{2}+\|\xi(t)\|_{X^{0}}^{2}+\left\|\xi_{x}(t)\right\|^{2} \leq C N^{2-2 r}
$$

$0 \leq t \leq T_{N}$, and thus

$$
\|\theta(t)\|+\|\xi(t)\| \leq C N^{1-r}
$$


for $0 \leq t \leq T_{N}$. Observe that due to $\theta \in S_{N}$,

$$
\|\theta(t)\|_{\infty} \leq C N^{\frac{1}{2}}\|\theta\| \leq C N^{\frac{1}{2}} N^{1-r} \leq C N^{\frac{3}{2}-r}<1
$$

for $N$ large enough and $0 \leq t \leq T_{N}$. Thus the maximality of $T_{N}$ is contradicted and we may take $T_{N}=T$. In this way, inequalities (5.21), (5.23) imply that

$$
\begin{aligned}
& \left\|\zeta(t)-\zeta_{N}(t)\right\|+\left\|u(t)-u_{N}(t)\right\| \leq\left\|\zeta(t)-P_{N} \zeta(t)\right\| \\
& \quad+\left\|P_{N} \zeta(t)-\zeta_{N}(t)\right\|+\left\|u(t)-P_{N} u(t)\right\|+\left\|P_{N} u(t)-u_{N}(t)\right\| \\
& =\|\rho(t)\|+\|\theta(t)\|+\|\sigma(t)\|+\|\xi(t)\| \leq C N^{1-r}
\end{aligned}
$$

for $0 \leq t \leq T$.

Remark 3. In the proof of Theorem 2, it is also important the presence of the dispersive terms of order $O\left(\epsilon^{2}\right)$ in system (5.9) to derive inequality (5.22) and apply Gronwall's lemma to deduce the error estimation.

\subsection{Numerical experiments}

We will check the numerical solver described above using an approximate solitary wave solution of system (5.1). Following the strategy in [8], let us suppose that the parameters $\alpha, \epsilon$ are small and

$$
\zeta=A_{1} u+A_{2} \alpha u^{2}+A_{3} \epsilon \mathcal{H}\left(u_{t}\right),
$$

where $A_{1}, A_{2}, A_{3}$ are constants to be calculated. Substituting into equations (5.1) and neglecting second-order terms $\alpha^{2}, \alpha \epsilon, \epsilon^{2}$, we have that

$$
\begin{aligned}
& A_{1} u_{t}+2 A_{2} \alpha u u_{t}+A_{3} \epsilon \mathcal{H}\left(u_{t t}\right)+2 \alpha A_{1} u u_{x}-u_{x}=0 \\
& u_{t}+\alpha u u_{x}+\left(1-\rho_{r}\right) A_{1} u_{x}+2 A_{2} \alpha\left(1-\rho_{r}\right) u u_{x}+\left(1-\rho_{r}\right) A_{3} \epsilon \mathcal{H}\left(u_{x t}\right) \\
& \quad=\rho_{r} \epsilon \mathcal{H}\left(u_{x t}\right) .
\end{aligned}
$$

Equation (5.25) implies that $u_{t}=\left(\rho_{r}-1\right) A_{1} u_{x}+O(\alpha, \epsilon)$. Therefore neglecting second-order terms, system (5.24)-(5.25) transforms into

$$
\begin{aligned}
& -A_{1} u_{t}+2 A_{2} \alpha\left(1-\rho_{r}\right) A_{1} u u_{x}+A_{3} \epsilon\left(1-\rho_{r}\right) A_{1} \mathcal{H}\left(u_{x t}\right)-2 \alpha A_{1} u u_{x}+u_{x} \\
& \quad=0 \\
& u_{t}+\alpha u u_{x}+\left(1-\rho_{r}\right) A_{1} u_{x}+2 A_{2} \alpha\left(1-\rho_{r}\right) u u_{x}+\left(1-\rho_{r}\right) A_{3} \epsilon \mathcal{H}\left(u_{x t}\right) \\
& \quad=\rho_{r} \epsilon \mathcal{H}\left(u_{x t}\right) .
\end{aligned}
$$

Multiplying equation $(5.26)$ by $A_{1}\left(1-\rho_{r}\right)$, it follows that

$$
\begin{aligned}
& -A_{1}^{2}\left(1-\rho_{r}\right) u_{t}+A_{1}\left(1-\rho_{r}\right) u_{x}+\left(2 A_{2} A_{1}^{2} \alpha\left(1-\rho_{r}\right)^{2}-2 \alpha A_{1}^{2}\left(1-\rho_{r}\right)\right) u u_{x} \\
& \quad+A_{3} A_{1}^{2}\left(1-\rho_{r}\right)^{2} \mathcal{H}\left(u_{x t}\right)=0, \\
& u_{t}+\alpha u u_{x}+\left(1-\rho_{r}\right) A_{1} u_{x}+\left(\alpha+2 A_{2} \alpha\left(1-\rho_{r}\right)\right) u u_{x} \\
& \quad+\left(\left(1-\rho_{r}\right) A_{3} \epsilon-\rho_{r} \epsilon\right) \mathcal{H}\left(u_{x t}\right)=0 .
\end{aligned}
$$


From these equations we conclude that

$$
\begin{aligned}
& -A_{1}^{2}\left(1-\rho_{r}\right)=1, \quad 2 A_{2} A_{1}^{2} \alpha\left(1-\rho_{r}\right)^{2}-2 \alpha A_{1}^{2}\left(1-\rho_{r}\right)=\alpha+2 A_{2} \alpha\left(1-\rho_{r}\right), \\
& A_{3} A_{1}^{2} \epsilon\left(1-\rho_{r}\right)^{2}=\left(1-\rho_{r}\right) A_{3} \epsilon-\rho_{r} \epsilon .
\end{aligned}
$$

Therefore

$$
A_{1}=-\frac{1}{\sqrt{\rho_{r}-1}}, \quad A_{2}=-\frac{1}{4\left(\rho_{r}-1\right)}, \quad A_{3}=\frac{\rho_{r}}{2\left(1-\rho_{r}\right)} .
$$

Substituting these values in equation (5.27), we obtain that

$$
u_{t}+\sqrt{\rho_{r}-1} u_{x}+\frac{3 \alpha}{2} u u_{x}-\frac{\rho_{r}}{2} \epsilon \mathcal{H}\left(u_{x t}\right)=0 .
$$

Observe that $u_{t}=-\sqrt{\rho_{r}-1} u_{x}+O(\alpha, \epsilon)$, and therefore we can change the derivative $u_{x t}$ by $u_{x x}$ in the equation above

$$
u_{t}+\sqrt{\rho_{r}-1} u_{x}+\frac{3 \alpha}{2} u u_{x}+\frac{\rho_{r}}{2} \epsilon \sqrt{\rho_{r}-1} \mathcal{H}\left(u_{x x}\right)=0 .
$$

Using that $u=-\sqrt{\rho_{r}-1} \zeta+O(\alpha, \epsilon)$, we further obtain an equation for the wave elevation

$$
\zeta_{t}+\sqrt{\rho_{r}-1} \zeta_{x}-\frac{3 \alpha}{2} \sqrt{\rho_{r}-1} \zeta \zeta_{x}+\frac{\rho_{r}}{2} \epsilon \sqrt{\rho_{r}-1} \mathcal{H}\left(\zeta_{x x}\right)=0
$$

It is well known that equation (5.28) has an exact solution in the form

$$
u(x, t)=a /\left(1+\left(\frac{x-c t-a_{0}}{\lambda}\right)^{2}\right)
$$

where

$$
\lambda=\frac{4 c_{2}}{a c_{1}}, \quad c=c_{0}+\frac{c_{1}}{4} a, \quad c_{0}=\sqrt{\rho_{r}-1}, \quad c_{1}=\frac{3 \alpha}{2}, \quad c_{2}=\frac{\rho_{r}}{2} \epsilon \sqrt{\rho_{r}-1} .
$$

Once the velocity is computed, the elevation is calculated as

$$
\zeta(x, t)=-\frac{1}{\sqrt{\rho_{r}-1}} u(x, t) .
$$

Benjamin-Ono equation (5.28) and its exact solitary wave solution (5.30) were introduced by Benjamin [6] (1967) and Ono [14] (1975).

In the first numerical experiment the computational domain is the interval $[0,100]$, the number of FFT points in the spatial domain is $2^{10}$ and $\Delta t=$ $40 / 4000$ is the time step. The model's parameters are $\alpha=\epsilon=0.01, \rho_{r}=1.5$ and $a=1$. To test the numerical method we will use the family of approximate solutions (5.30)-(5.31) of system (5.1). Thus we solve numerically system (5.1) with the following initial conditions for $u, \zeta$ :

$$
u(x, 0)=\frac{a}{1+\left(\frac{x-a_{0}}{\lambda}\right)^{2}}, \quad \zeta(x, 0)=-\frac{1}{\sqrt{\rho_{r}-1}} u(x, 0) .
$$



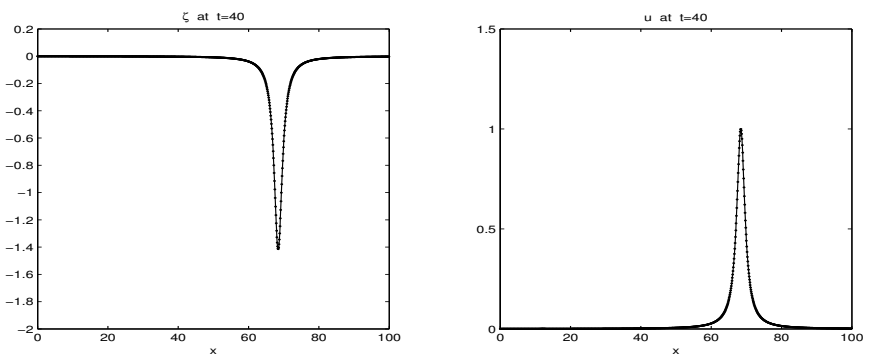

Figure 2. In points: approximate solitary wave solution (5.30)-(5.31). In solid line: solution $\left(\zeta_{\text {num }}, u_{\text {num }}\right)$ obtained with the numerical solver.
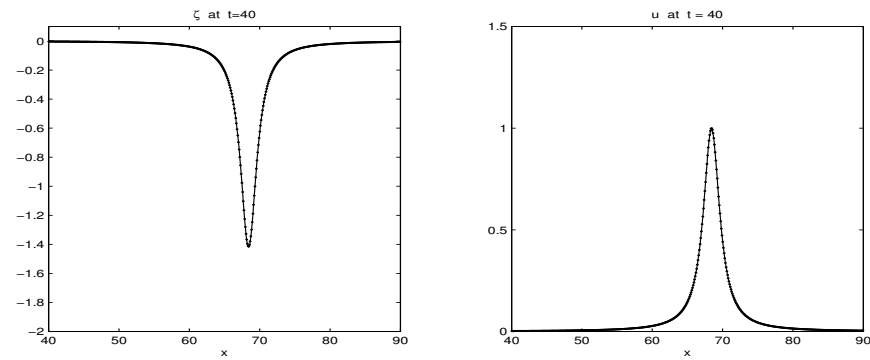

Figure 3. Zoom of Figure 2. In points: approximate solitary wave solution (5.30)-(5.31). In solid line: solution $\left(\zeta_{\text {num }}, u_{\text {num }}\right)$ obtained with the numerical solver.

We point out that the parameter $a_{0}$ controls the initial position of the solitary wave. In Figure 2 we compare the numerical solution with the approximations (5.30)-(5.31) computed at time $t=40$. In Figure 3 we show a zoom of Figure 2. We see that they perfectly agree with good accuracy and neither numerical dispersion nor dissipation are evidenced in the profiles of the fluid velocity and wave elevation. The wave speed is approximately $c \approx 0.7109$ and the initial position is $a_{0}=40$.

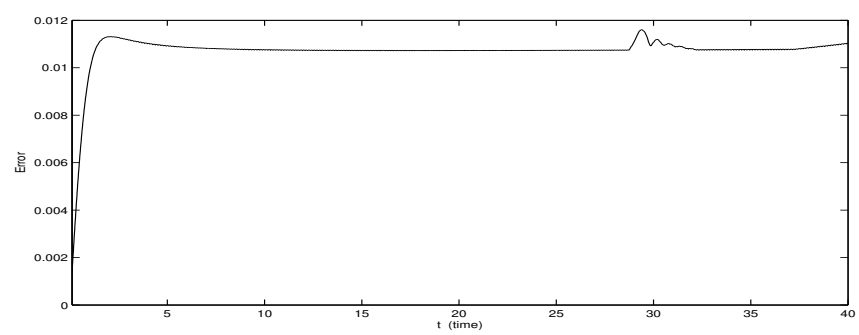

Figure 4. $E(t)=\left\|\zeta(t)-\zeta_{\text {num }}(t)\right\|_{\infty}+\left\|u(t)-u_{\text {num }}(t)\right\|_{\infty}$ as a function of time.

In Figure 4 we show the difference in the supremum norm between the numerical solution and the approximate solitary wave solution given in (5.30)- 
(5.31). This experiment is in accordance with the convergence results established in this paper.
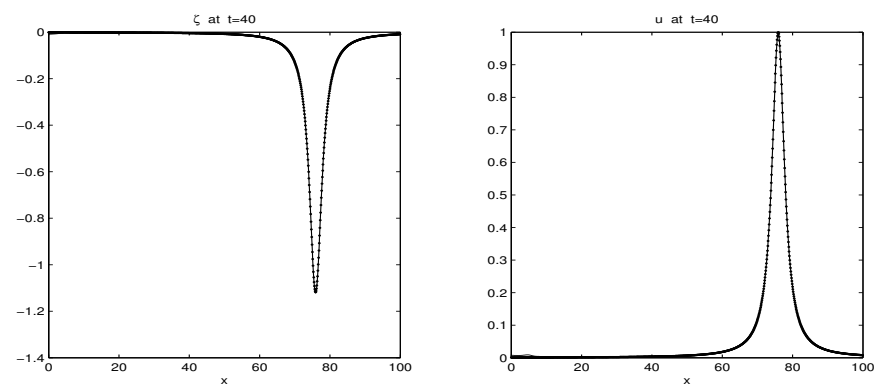

Figure 5. In points: approximate solitary wave solution (5.30)-(5.31). In solid line: solution $\left(\zeta_{\text {num }}, u_{\text {num }}\right)$ obtained with the numerical solver.
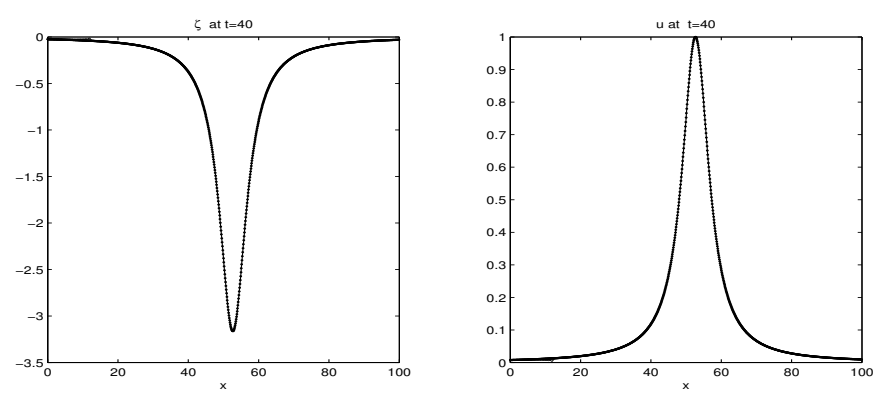

Figure 6. In points: approximate solitary wave solution (5.30)-(5.31). In solid line: solution $\left(\zeta_{\text {num }}, u_{\text {num }}\right)$ obtained with the numerical solver.

In Figure 5 we show a new numerical experiment using the same parameters as before but with $\rho_{r}=1.8$. In Figure 6 is displayed a numerical experiment in the case that $\alpha=0.001, \epsilon=0.01$ and $\rho_{r}=1.1$.

In Figure 7 we show a numerical experiment showing the effect of the dispersive terms of order $O\left(\epsilon^{2}\right)$ on solutions of system (1.1). In this case $\alpha=0.001$, $\epsilon=0.01, \rho_{r}=1.1$ and the initial pulse is a Gaussian disturbance in the form

$$
\zeta(x, 0)=\exp \left(-\sigma\left(x-a_{0}\right)^{2}\right), \quad u(x, 0)=\exp \left(-\sigma\left(x-a_{0}\right)^{2}\right)
$$

with $\sigma=100$ and $a_{0}=40$. Observe a discrepancy between the amplitudes of the oscillatory parts of wave profiles. Finally in Figure 8 we repeat the previous experiment but using the parameters $a_{0}=100, \alpha=0.01, \epsilon=0.1, \rho_{r}=1.8$ and the computational domain is $[0,200]$. We observe that the discrepancy between the two profiles is more appreciable than in the previous simulation.

The last two experiments corroborate numerically that the terms of order $O\left(\epsilon^{2}\right)$ are corrections of previous models for the internal wave problem considered which alter the dispersive-wave mechanism and become important in 

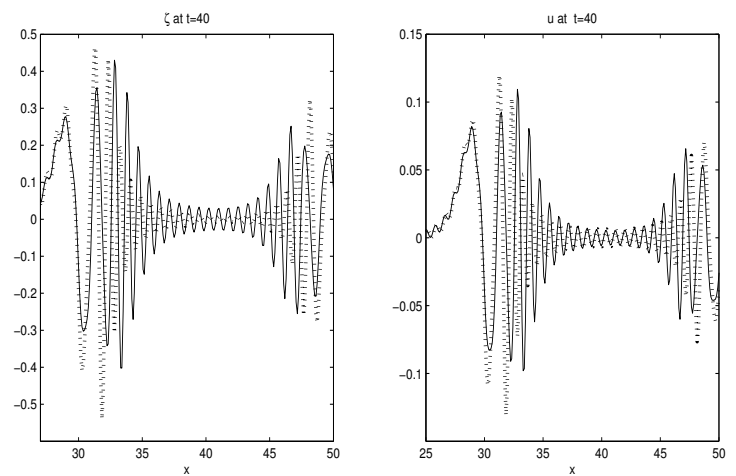

Figure 7. In points: numerical solution of system (1.1) without terms of order $O\left(\epsilon^{2}\right)$. In solid line: numerical solution of full system (1.1).
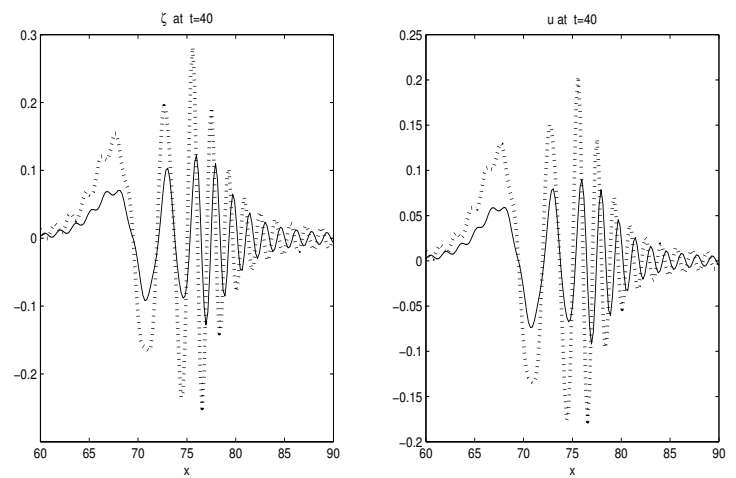

Figure 8. In points: numerical solution of system (1.1) without terms of order $O\left(\epsilon^{2}\right)$. In solid line: numerical solution of full system (1.1).

predicting the propagation of internal water waves in the physical regime analyzed by the present paper.

\section{Conclusions}

We introduced a new model for the propagation of an internal wave at the interface of two immiscible fluids with constant densities, contained at rest in a long channel with horizontal rigid top and bottom. This system includes dispersive terms of second order $O\left(\epsilon^{2}\right)$ which were neglected in previous works for different regimes of the problem. These dispersive-type terms allowed us to employ the Banach fixed point theorem to establish existence and uniqueness of solutions of system (1.1). Other important property of the particular formulation (1.1) is the fact that their solutions can be bounded in terms of the initial data, enabling the use of classical techniques of functional analysis to extend them for any time $t>0$. Furthermore, the new formulation (2.31)- 
(2.32) was stated in terms of the fluid velocity at a fixed depth $Z_{0}$, which is easier of measuring than the commonly used depth-averaged velocity employed for instance in $[8,9]$. Regarding possible engineering applications, this is an important advantage of the family of systems (2.31)-(2.32). We also introduced a Galerkin spectral numerical scheme to approximate the solution of the corresponding initial value problem and established a theory of convergence of the semidiscrete numerical scheme for the spatial periodic case. The fact that no differentiation matrices are used in this scheme makes the implementation easier than in [8] where a pseudospectral scheme was applied to system (1.1) neglecting terms of order $O\left(\epsilon^{2}\right)$.

\section{References}

[1] L. Abia and J. Sanz-Serna. The spectral accuracy of a full-discrete scheme for a nonlinear third-order equation. Computing, 44:187-196, 1990. http://dx.doi.org/10.1007/BF02262215.

[2] C.T. Anh. Influence of surface tension and bottom topography on internal waves. Math. Models Methods Appl. Sci., 19(12):2145-2175, 2009. http://dx.doi.org/10.1142/S0218202509004078.

[3] C.T. Anh. On the Boussinesq/Full dispersion systems and Boussinesq/Boussinesq systems for internal waves. Nonlinear Anal., 72:409-429, 2010. http://dx.doi.org/10.1016/j.na.2009.06.076.

[4] U.M. Ascher, S.J. Ruuth and B.T.R. Wetton. Implicit-explicit methods for timedependent partial differential equations. SIAM J. Numer. Anal., 32(3):797-823, 1995. http://dx.doi.org/10.1137/0732037.

[5] C. Basdevant, M. Deville, P. Haldenwang, J. Lacroix, J. Quazzani, R. Peyret, P. Orlandi and A. Patera. Spectral and finite difference solutions of the Burgers equation. Comput. Fluids, 14:23-41, 1986. http://dx.doi.org/10.1016/0045-7930(86)90036-8.

[6] T.B. Benjamin. Internal waves of permanent form in fluids of great depth. $J$. Fluid Mech., 29:559-562, 1967. http://dx.doi.org/10.1017/S002211206700103X.

[7] J.L. Bona, D. Lannes and J.C. Saut. Asymptotic models for internal waves. J. Math. Pures Appl., 89(9):538-566, 2008. http://dx.doi.org/10.1016/j.matpur.2008.02.003.

[8] W. Choi and R. Camassa. Weakly nonlinear internal waves in a two-fluids system. J. Fluid Mech., 313:83-103, 1996. http://dx.doi.org/10.1017/S0022112096002133.

[9] W. Choi and R. Camassa. Fully nonlinear internal waves in a two-fluid system. $J$. Fluid Mech., 396:1-36, 1999. http://dx.doi.org/10.1017/S0022112099005820.

[10] J. Garnier, R. Kraenkel and A. Nachbin. Optimal Boussinesq model for shallowwater waves interacting with a microstructure. Phys. Rev. E, 76:046311, 2007. http://dx.doi.org/10.1103/PhysRevE.76.046311.

[11] J.C. Muñoz Grajales. Decay of solutions of a Boussinesq-type system with variable coefficients. Waves Random Complex Media, 22(4):589-612, 2012. http://dx.doi.org/10.1080/17455030.2012.734641. 
[12] J.C. Muñoz Grajales and A. Nachbin. Improved Boussinesq-type equations for highly-variable depths. IMA J. Appl. Math., 71:600-633, 2006. http://dx.doi.org/10.1093/imamat/hxl008.

[13] O. Nwogu. Alternative form of Boussinesq equations for nearshore wave propagation. J. Waterway, Port, Coastal Ocean Eng., 119(6):618-638, 1993. http://dx.doi.org/10.1061/(ASCE)0733-950X(1993)119:6(618).

[14] H. Ono. Algebraic solitary waves in stratified fluids. J. Phys. Soc. Japan, 39:1082-1091, 1975. http://dx.doi.org/10.1143/JPSJ.39.1082.

[15] J.E. Pasciak. Spectral and pseudo-spectral methods for advection equation. Math. Comput., 35:1081-1092, 1980.

[16] B. Pelloni and V.A. Dougalis. Error estimates for a fully discrete spectral scheme for a class of nonlinear, nonlocal dispersive wave equations. Appl. Numer. Math., 37:95-107, 2001. http://dx.doi.org/10.1016/S0168-9274(00)00027-1.

[17] A. Ruiz de Zárate and A. Nachbin. A reduced model for internal waves interacting with topography at intermediate depth. Commun. Math. Sci., 6(2):385-396, 2008. http://dx.doi.org/10.4310/CMS.2008.v6.n2.a6.

[18] A. Ruiz de Zárate, D.G. Alfaro Vigo, A. Nachbin and W. Choi. A higher-order internal wave model accounting for large bathymetric variations. Stud. Appl. Math., 122:275-294, 2009. http://dx.doi.org/10.1111/j.14679590.2009.00433.x.

[19] V. Tomée and A.S. Vasudeva Murphy. A numerical method for the periodic Benjamin-Ono equation. BIT, 38:597-611, 1998. http://dx.doi.org/10.1007/BF02510262.

[20] G.B. Whitham. Linear and Nonlinear Waves. John Wiley, New York, London, Sidney, 1974. 